\title{
NPP VIIRS Geometric Performance Status
}

\author{
Guoqing (Gary) Lin*a, ${ }^{* a}$, Robert E. Wolfe ${ }^{\mathrm{b}}$, Masahiro Nishihama, ${ }^{\mathrm{c}, \mathrm{b}}$ \\ ${ }^{a}$ Innovim, 7501 Greenway Center Drive, Suite 660, Greenbelt, MD, USA 20770; \\ bNASA Goddard Space Flight Center, Code 614.5, 8800 Greenbelt Rd, Greenbelt, MD, USA 20771; \\ 'Sigma Space Corporation, 4600 Forbes Blvd, Lanham, MD, USA 20706
}

\begin{abstract}
Visible Infrared Imager Radiometer Suite (VIIRS) instrument on-board the National Polar-orbiting Operational Environmental Satellite System (NPOESS) Preparatory Project (NPP) satellite is scheduled for launch in October, 2011. It is to provide satellite measured radiance/reflectance data for both weather and climate applications. Along with radiometric calibration, geometric characterization and calibration of Sensor Data Records (SDRs) are crucial to the VIIRS Environmental Data Record (EDR) algorithms and products which are used in numerical weather prediction (NWP). The instrument geometric performance includes: 1) sensor (detector) spatial response, parameterized by the dynamic field of view (DFOV) in the scan direction and instantaneous FOV (IFOV) in the track direction, modulation transfer function (MTF) for the 17 moderate resolution bands (M-bands), and horizontal spatial resolution (HSR) for the five imagery bands (I-bands); 2) matrices of band-to-band co-registration (BBR) from the corresponding detectors in all band pairs; and 3) pointing knowledge and stability characteristics that includes scan plane tilt, scan rate and scan start position variations, and thermally induced variations in pointing with respect to orbital position. They have been calibrated and characterized through ground testing under ambient and thermal vacuum conditions, numerical modeling and analysis. This paper summarizes the results, which are in general compliance with specifications, along with anomaly investigations, and describes paths forward for characterizing on-orbit BBR and spatial response, and for improving instrument on-orbit performance in pointing and geolocation.
\end{abstract}

Keywords: NPP, VIIRS, FOV, MTF, HSR, BBR, pointing, geolocation

\section{INTRODUCTION}

The VIIRS instrument design draws from heritage moderate resolution instrument designs with a few unique features. The instrument scans the earth in the cross-track direction by using a half angle mirror (HAM) to de-rotate the incoming rays from the rotating telescope assembly (RTA) into a fixed aft-optics assembly (AOA), that includes a visible and near infrared (VisNIR) focal plane assembly (FPA), a short- and mid-wave infrared (SWMWIR) FPA and a long-wave infrared (LWIR) FPA in a cold dewar, ${ }^{1,2}$ (Figure 1). The physical layout of these FPAs and bands within them is schematically shown in Figure $2^{1,2}$. There are 17 moderate resolution bands (M-bands) (of which bands M16A and M16B are the same in spectral range and will be used as one band on the ground by averaging radiometric responses), five imagery bands (I-bands) and a day-night band (DNB). (The DNB is not the focus of this paper and thus not discussed further.) Each M-band has 16 detectors and each I-band has 32 detectors. These detectors are rectangular with the smaller dimension in the along scan direction. An aggregation scheme of $3 \times 1,2 \times 1$ and $1 \times 1$ respectively in the nadir, the middle and the edge of scan is employed to limit the growth of pixel in the scan direction. Table 1 lists the I-bands horizontal sampling intervals (HSIs) on earth surface in the three aggregation zones, at the nominal satellite altitude of $824 \mathrm{~km}$, earth radius of $6378 \mathrm{~km}$ at the equator, scan rate of $3.531 \mathrm{rad} / \mathrm{s}$, un-aggregated angular sampling interval (ASI) of $155.83 \mu \mathrm{rad}$ and track ASI of $455.5 \mu \mathrm{rad}$. The HSIs for M-bands are twice of those for I-bands. Also, a bow-tie deletion scheme is used to trim 1 (2) and 2 (4) M (I) band detectors at the scan edges in the $2 \times 1$ and $1 \times 1$ aggregation zones, respectively. These two geometric features are unique comparing to legacy instruments such as the Advanced Very High Resolution Radiometer (AVHRR) and the Moderate Resolution Imaging Spectroradiometer (MODIS) ${ }^{1}$.

*Gary.Lin@nasa.gov; glin@innovim.com; phone 301 614-5451; fax 301 614-5269; innovim.com 
Table 1. Nominal I-band HSI at the ends of the three aggregation zones

\begin{tabular}{|c|c|c|c|c|c|c|}
\hline & \multicolumn{2}{|c|}{ Aggregation 3 Zone } & Aggregation 2 Zone & \multicolumn{2}{c|}{ Aggregation 1 Zone } \\
\hline & Nadir & End & Start & End & Start & End of scan \\
\hline Scan Angle (Deg) & 0 & \pm 31.72 & \pm 31.72 & \pm 44.86 & \pm 44.86 & \pm 56.28 \\
\hline HSI_scan (m) & 385 & 577 & 385 & 644 & 322 & 822 \\
\hline HSI_track (m) & 367 & 443 & 443 & 556 & 556 & 807 \\
\hline
\end{tabular}

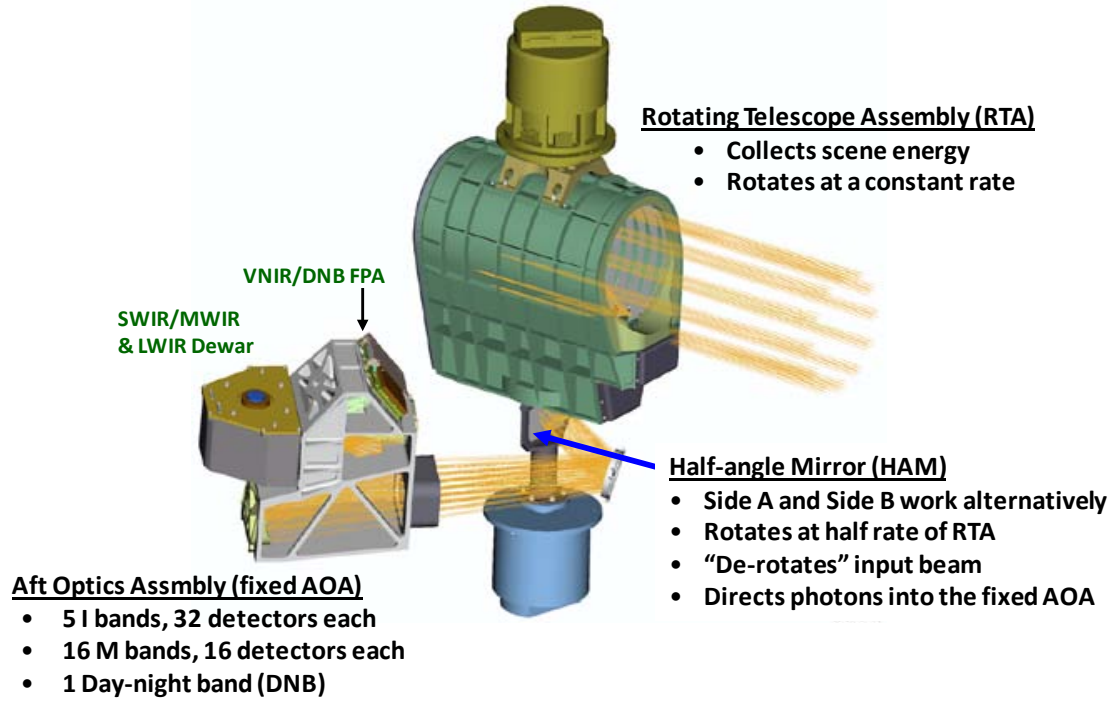

Figure 1. VIIRS optical system

Nominal Timing Offsets (in Moderate Band Sampling Interval units) Relative to Focal Plane Reference Axis
20
15
10
5
$\mathbf{0}$
$-5$
$-10-15$
$-20$

VIS/NIR

FPA

DNB

$\begin{array}{lllllllll}\text { M6 } & \text { M5 } & \text { M7 } & \text { I2 } & \text { I1 } & \text { M3 } & \text { M4 } & \text { M2 } & \text { M1 }\end{array}$

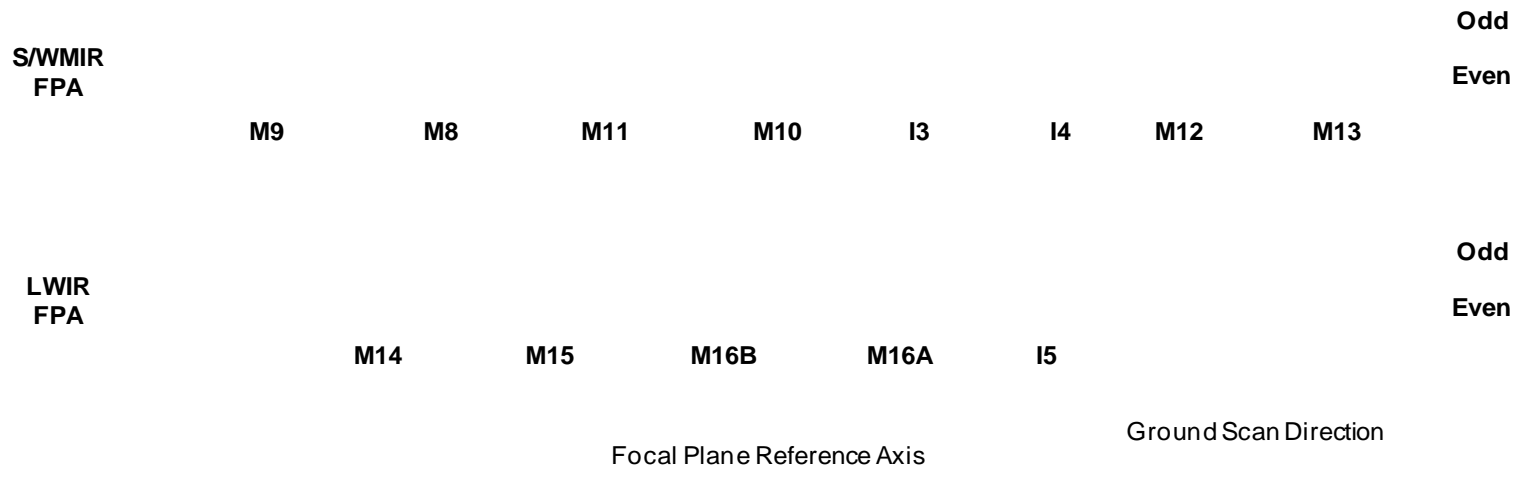

Figure 2. VIIRS focal plane assemblies and band layout in the scan direction ${ }^{1}$.

A VIIRS engineering development unit (EDU) was first developed and tested and subsequently a first flight unit (F1) was built for the NPP satellite. Ground tests and modeling and analysis included the instrument spectral response, 
radiometric response and geometric response. This paper focuses on the geometric calibration and characterization. Section 2 details spatial response, parameterized by the dynamic field of view (DFOV) in the scan direction and instantaneous FOV (IFOV) in the track direction, modulation transfer function (MTF) for M-bands, and horizontal spatial resolution (HSR) for I-bands. Section 3 reports the test results in band-to-band co-registration (BBR) between all band pairs, including nested I-bands within the M-bands. Section 4 describes pointing characteristics from ground measurements and numerical modeling using a structural, thermal and optical performance (STOP) model. The results are compared with sensor specification ${ }^{3}$ for performance compliance. The last Section concludes the paper with a description of the path forward for characterizing on-orbit BBR and spatial response, and for improving instrument onorbit performance in pointing and geolocation.

The pre-launch geometric results reported here are primarily based on our modeling and analysis using ground test and model data provided by the instrument vendor (Raytheon). These results are similar to those reported by other government and contractor teams (see Acknowledgements).

\section{VIIRS SENSOR SPATIAL RESPONSE PERFORMACE}

The instrument sensor (detector) spatial response is system spatial impulse response for each detector as measured by its point spread functions (PSFs). Physically, a PSF is a weighting function for a detector to collect energy (as converted to a digital number: $d n$ ) from a scene $E(x, y)$. Their relationship may be expressed as a convolution

$$
d n\left(x_{S}, y_{D}\right)=c E(x, y) \otimes P S F(x, y)=c \oiint_{\left(x^{\prime}, y^{\prime}\right) \in R} E\left(x^{\prime}, y^{\prime}\right) \operatorname{PSF}\left(x_{S}-x^{\prime}, y_{D}-y^{\prime}\right) d x^{\prime} d y^{\prime}
$$

where $\left(x_{S}, y_{D}\right)$ is the pointing location of a sample for the detector, and $c$ is a lumped coefficient for other factors such as the instrument spectral and radiometric responses. In the ground tests, the PSF for each detector is decomposed into and measured by line spread functions (LSFs) in the scan and track directions. Both LSFs are measured using special test equipment (STE) that includes a light source, an integrating sphere, reticles with various slit configurations and a three mirror collimator (TMC) that is used to focus the light into the static (track direction) or scanning (scan direction) sensor ${ }^{4}$. The mathematical principle of the LSF measurement is the identity result of a distribution convolving with a Dirac delta distribution (which could be viewed physically as a normalized ideal line source) ${ }^{5,6}$, i.e.

$$
\operatorname{LSF}\left(x_{o}\right)=\operatorname{LSF}(x) \otimes \delta(x), \quad \delta(x)=1 @ x_{o} \text { only }
$$

where the location $x_{o}$ provides desired phase relative to the detector's line of sight (LOS) in terms of ASI.

The LSFs are then parameterized by the DFOV in the scan direction and the IFOV in the track direction at the full width of half maximum (FWHM) for all bands, the MTF for M-bands and the HSR for I-bands, and compared against sensor specification $^{3}$. The MTF is a function of spatial frequency $(f)$ and is defined as the magnitude of the normalized Fourier transform $(F)$ of the LSF so that it is equal to 1 at the origin, that is:

$$
\operatorname{MTF}(f)=\frac{|F(L S F)|}{|F(L S F)|_{\mid f=0}} .
$$

The spatial frequency is expressed in unit of Nyquist Frequency, i.e.:

$$
f_{\text {Nyquist }}=\left(\frac{1}{2 H S I}\right) \text {. }
$$

The horizontal spatial resolution (HSR) is defined as one-half the longest spatial wavelength on the earth surface at which the MTF drops to 0.5, i.e.:

$$
\operatorname{MTF}\left(\frac{1}{2 H S R}\right)=0.5
$$


Similar to the HSI, the HSR varies with scan angle from nadir. In the test data analysis, we use angular spatial resolution (ASR) directly, without converting it back and forth to HSI and HSR.

The sensor specification places the spatial response requirements in the un-aggregated zone, except for an HSR requirement at nadir. The track IFOV is specified at 445 and $891 \mu \mathrm{rad}$ with 5\% margin for I- and M-bands, respectively. The scan DFOV is specified at 123 and $393 \mu$ rad with $10 \%$ margin for I- and M-bands, respectively. The MTF for Mbands is specified to be greater than $0.3,0.5,0.7$ and 0.9 at $1.0,0.75,0.5$ and 0.25 Nyquist frequencies, respectively. The HSR for I-bands is specified to be less than $400 \mathrm{~m}$ at the nadir and $800 \mathrm{~m}$ at the end of scan (EOS). When assessing performance compliance, we convert the FOV specification in terms of ASI and the HSR specification in terms of HSI at the nadir or at the EOS.

\subsection{Track static LSF measurements and test data analysis}

The setup for measuring the track static LSFs is to use a fold mirror in the TMC to project multiple parallel slit sources into the locked RTA and steers the mirror in equal sized steps to get the LSF values at different $x_{o}$ phases as expressed in Eq. (2). Physically, the "delta function" has a finite slit width, so that the measurements result in a combined aperture response functions (CARF) ${ }^{7,8,9}$, which has tapered leading and trailing edges, as shown in Figure 3(a). The size of edge tapering is the width of the reticle slits used in the test, which are about $12 \%$ of the ASI for M-band and $24 \%$ of the ASI for I-bands. The CARF is used as a proxy for the true detector LSF for sensor spatial response parameterization. The artifact will not affect IFOV parameterization from the CARF full width half maximum (HWHM) in the ideal case of a rectangular LSF. It does cause an under-estimate of the MTF and an over-estimate of the HSR. So, these are more conservative estimates than those from a true LSF characterization.

The complete track static LSFs were measured under the ambient condition. Limited tests were conducted under thermal vacuum (TVac) conditions for a few detectors in band M12. Figure 3(b) shows the track LSFs for M12 detectors 1, 12 and 13 under ambient condition, TVac nominal performance plateau and cold operational plateau, where Detector 1 FWHMs (their LSFs are in blue lines) are consistently narrower than others, which is also shown in Figure 4. The root cause has been theorized as an anomaly in the micro-lenses tolerance. Different thermal conditions seem to also affect the shapes of the M12 LSFs.

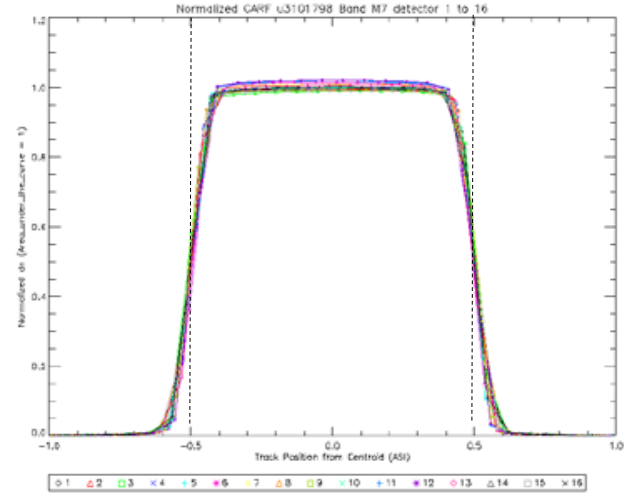

(a)

Figure 3. Example VIIRS LSFs: (a) Track static "nominal” LSFs for all detectors in band M7; (b) Anomalous LSFs for M12 detectors 1, 12 and 13.

The track detector spatial response parameters, the IFOV for all bands, the MTF for M-bands and the HSR at the end of scan (EOS) for I-bands under ambient conditions tests are computed and shown respectively in Figures 4, 5 and 6 . In general, the results meet the original specification, except for the IFOV where a known under-performing detector \#1 in band M12 and a few detectors in band I5. For these under-performing detectors, a "wavier" was submitted against the IFOV specification, loosening the performance compliance criteria. 


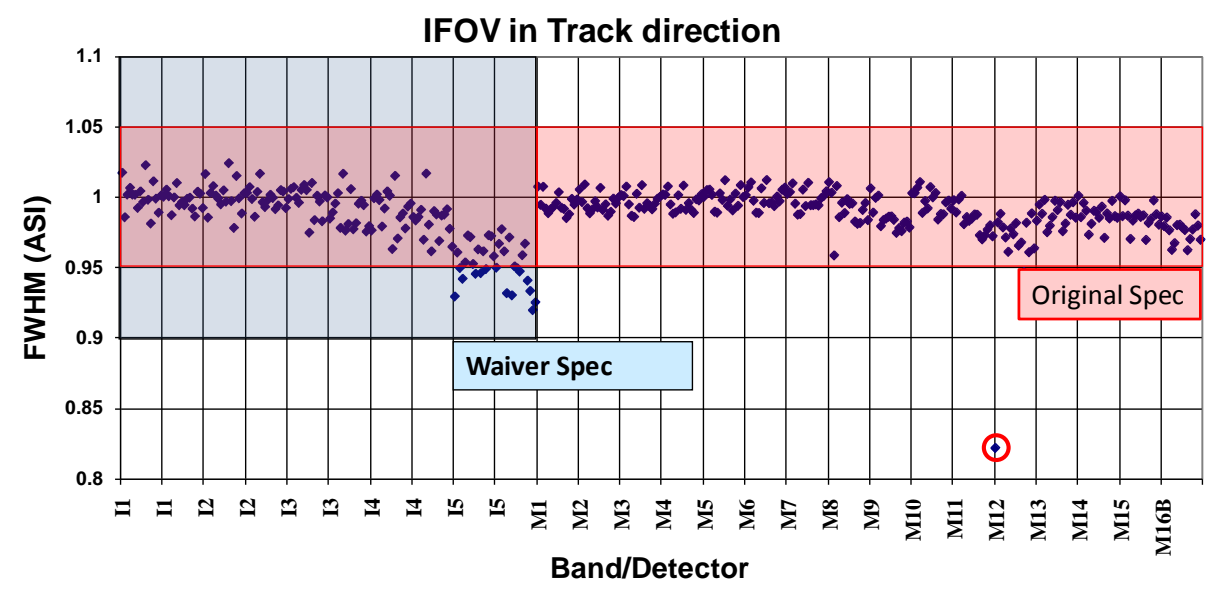

Figure 4. Ambient test result of I- and M-bands track IFOV. Each point represents an individual detector.

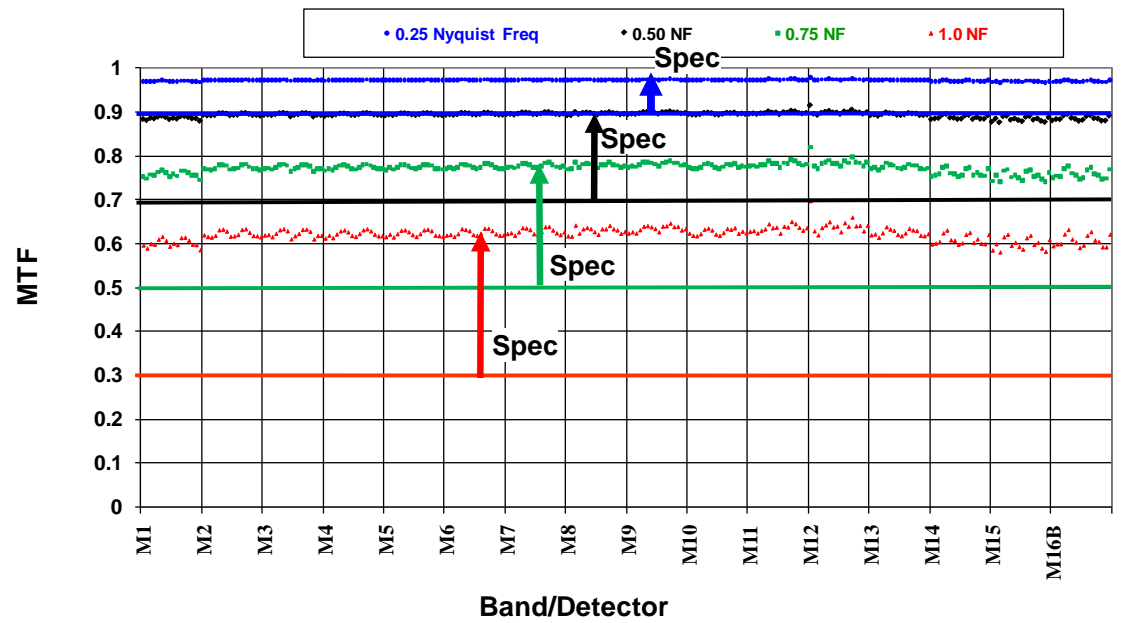

Figure 5. Ambient test result of the M-bands' MTF in track direction at 0.25, 0.5, 0.75 and 1.0 Nyquist frequencies (the straight lines denote the minimum requirements and the arrows denote the direction of compliance).

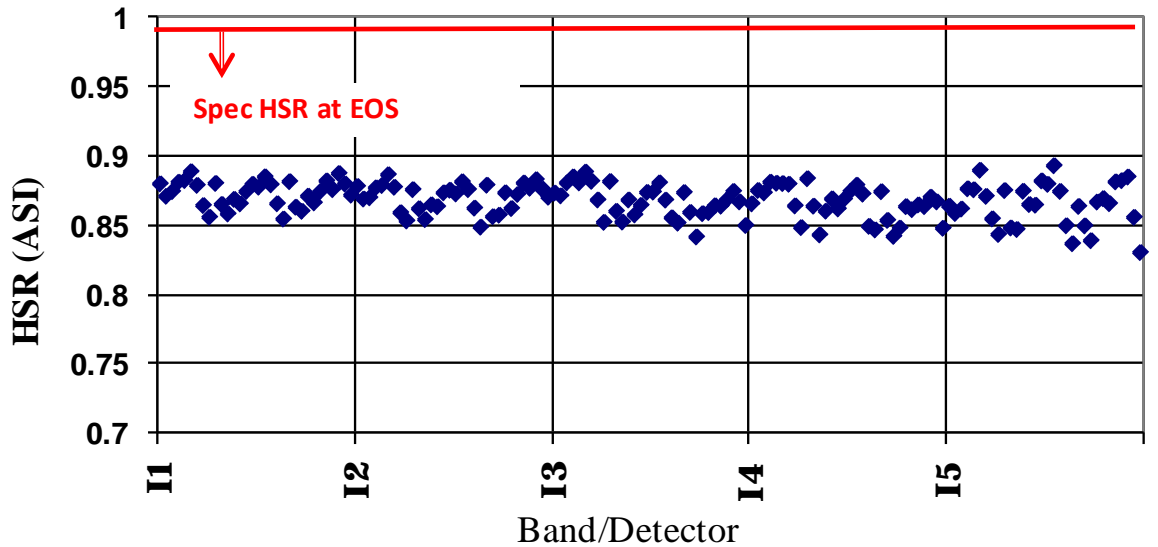

Figure 6. Ambient test result of the I-bands' HSR (the straight lines denote the minimum requirements and the arrow denotes the direction of compliance). 


\subsection{Scan dynamic LSF measurements and test data analysis}

During a sampling interval, the VIIRS telescope rotates an angular sampling interval (ASI) in the scan direction, the majority of which is the sampling integration time (D), with a small portion of the time (and corresponding travel angle) for signal read-out and electronic reset. When a detector's static or instantaneous LSF (ILSF) convolves with the integration drag, a dynamic LSF (DLSF) is formed. This process is schematically illustrated in Figure 7. Note that the shape of the ILSF and the corresponding IFOV are not known. The design of the VIIRS places the signal readout and reset time before sampling integration. The pointing, and the corresponding geolocation of the sample, is at the middle of the sampling integration, which we call the dynamic line of sight (DLOS) in the scan direction. In the track direction, however, during the integration time, the ILSF only moves with the spacecraft, which is negligibly slow compared to the movement in the scan direction. Thus, the resultant LSF in the track direction remains as ILSF. Because of this difference, they are measured with a different setup on the ground, as described in the previous Section for track ILSF and below for the scan DLSF.

For VIIRS, the scan DLSF was measured using a reticle with 11 phased slits ${ }^{4}$ perpendicular to the scan direction. The TMC was used to project images of these slits into the scanning RTA. The configuration of the slits is such that each slit samples the DLSF value at different $x_{o}$ phase as expressed in Eq. (2). The intent of the setup was that the 11 slits were to sample the DLSF in equal spacing in fractional ASIs after being shifted by their phase difference, and that all 11 slits have equal throughput. These assumptions were proven false in the ground testing, with "vertical banding" caused by slit throughput non-uniformity and "horizontal banding" caused by slit phase non-uniformity, as shown in Figure 8(a). Fortunately, the telescope rotated 100 times $^{4}$ in each DLSF ground testing, giving 100 samples for each slit. Furthermore, the synchronization of RTA and HAM at each scan start resulted in scan start position variation of about one half ASIs for I-band DLSFs and a quarter ASIs for M-band DLSFs (e.g., Figure 17). We made use of the variations to iteratively adjust the slit phases and re-normalize the slit throughputs, yielding smooth DLSF for each detector, as shown for an example in Figure 8(b).

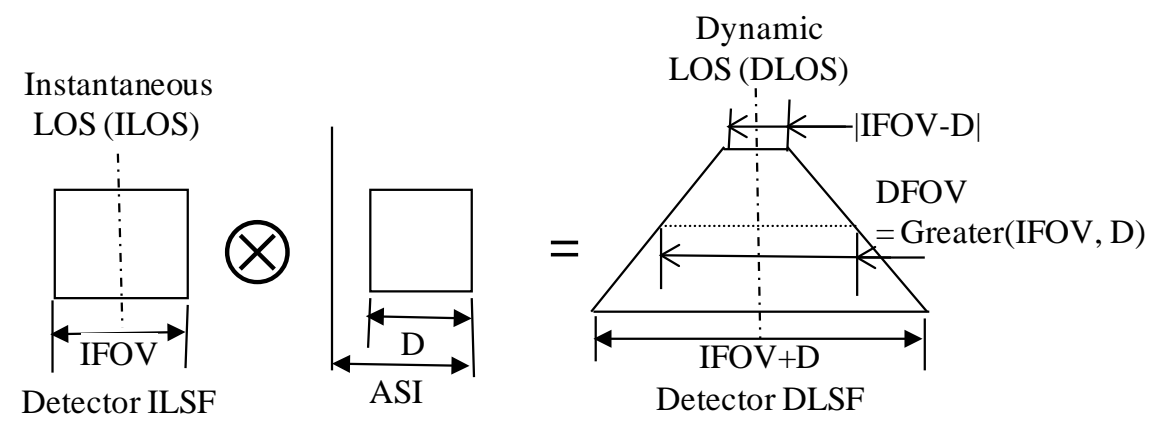

Figure 7. In the scan direction, the DLSF is a convolution of the detector ILSF with the integration drag.

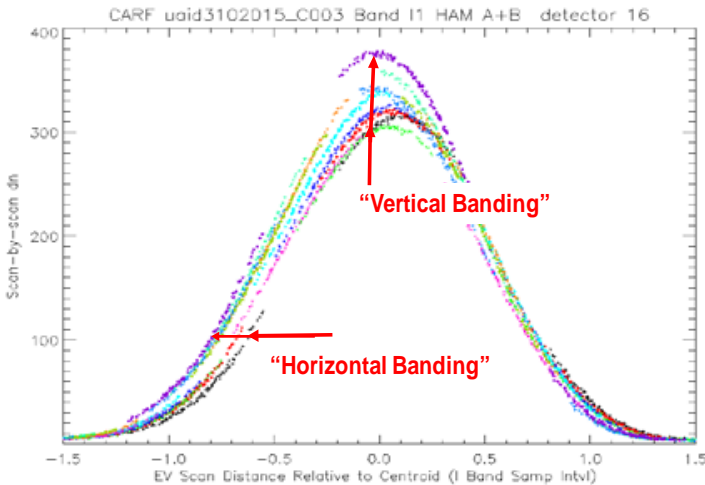

(a)

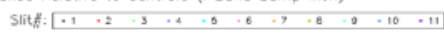

(b)

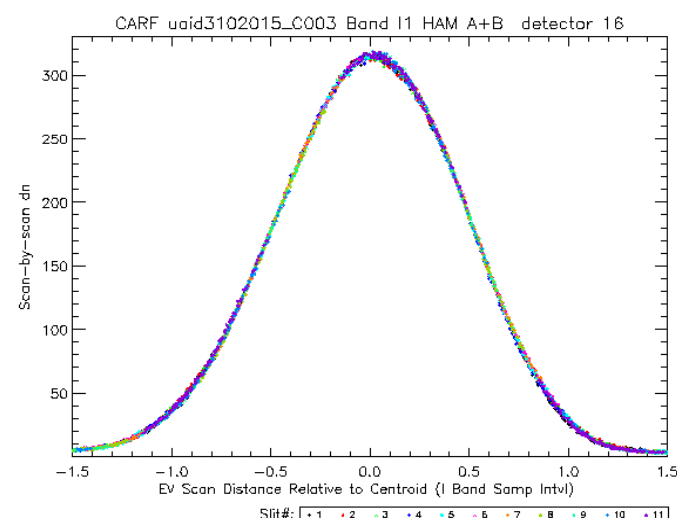

Figure 8. Scan direction DLSF for band I1 detector 16: (a) Original measurement with throughput and slit phase artifacts; (b) With throughput and phase corrections. 
The scan detector spatial response parameters, the DFOV, the MTF and the HSR, are then computed from the smooth DLSFs and shown respectively in Figures 9, 10 and 11 from the complete tests under thermal vacuum (TVac) cold and nominal performance plateaus. As we can see, the sensor specification has evolved over time. The original specification was based on ideal optical performance of the instrument design with some margins, which is demonstrated in the track IFOVs for all bands when their detector sizes are relatively large (Figure 4). The M-bands detectors are also relatively large in the scan direction, so the scan DFOV meets original specification for a majority of the detectors. However, for the I-bands, the detector size and field stop in the scan direction are relatively small. The instrument effective focal length (EFL) and non-ideal focus plays a significant role, so that the DFOVs are out-of-bounds of the original specification. The test results meet the waiver specification, which is reasonable as they are still within the pixel size of ASI in the scan direction.

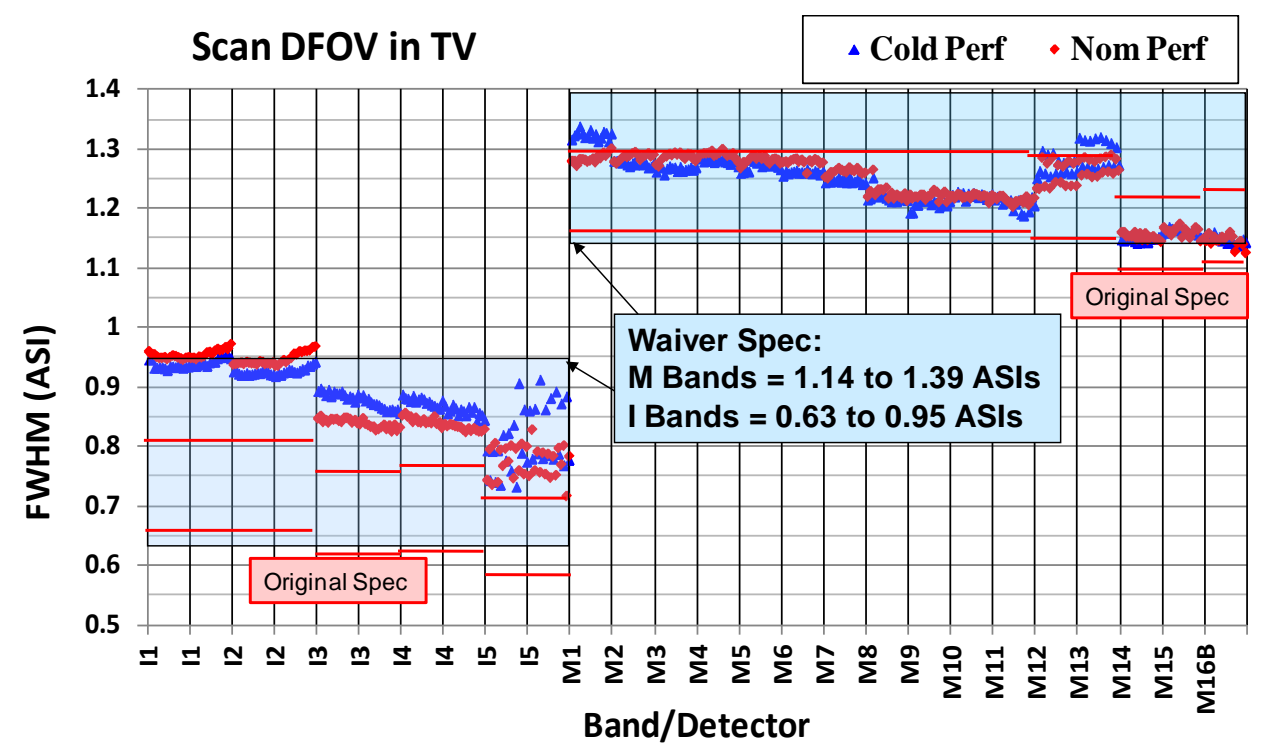

Figure 9. The I- and M-bands DFOV from TVac cold and nominal performance plateaus.

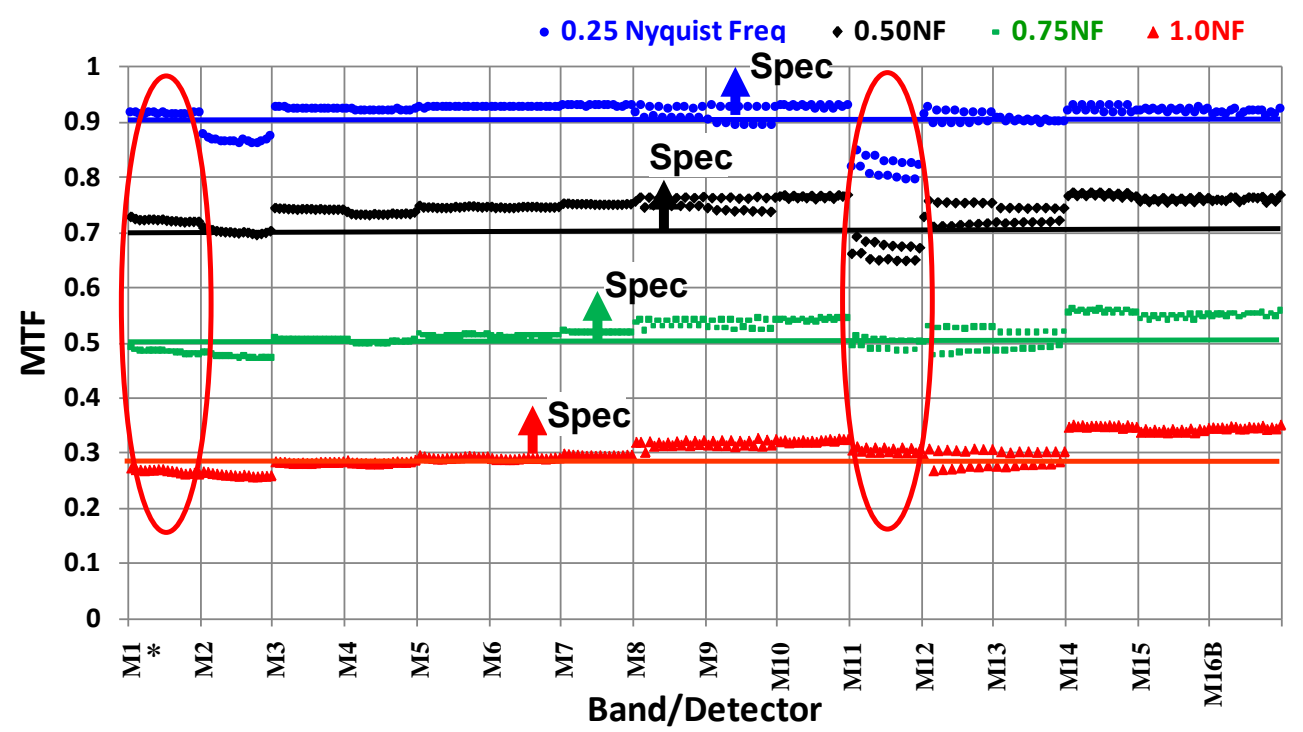

Figure 10. The M-bands scan MTF from TVac nominal performance plateau (M1* data is from a special test at hot performance plateau, see Figure 12(a)). 


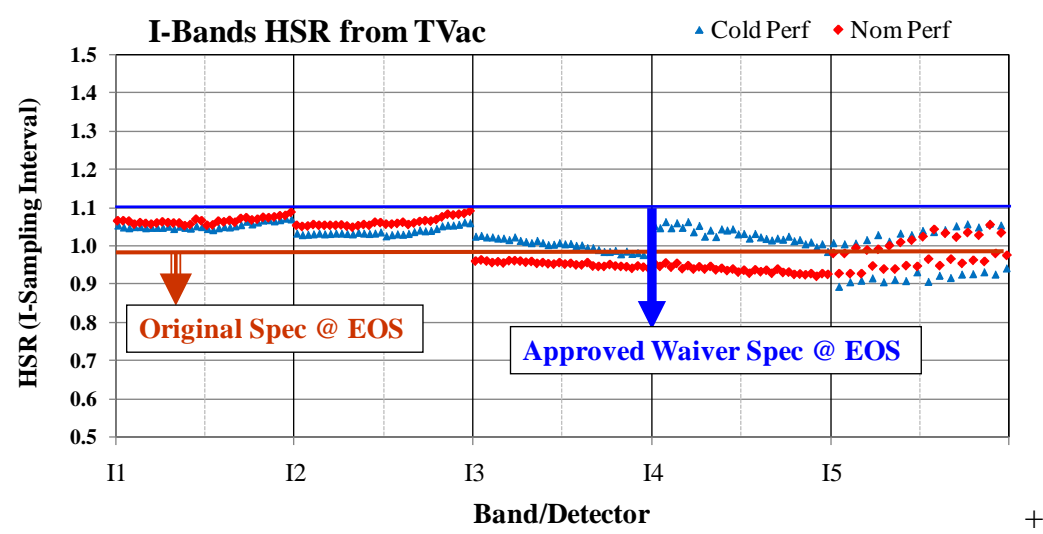

Figure 11. The I-bands scan HSR at the end of scan (EOS) from TVac cold and nominal performance plateaus.

For the M-bands MTF in the scan direction, the test results in the TVac nominal performance plateau meet requirements for a majority of bands/detectors, as shown in Figure 10 (the results from TVac cold performance plateau are not shown here but are similar to those at the nominal performance plateau). There are two classes of significant exceptions: 1) Band M1 DLSF has an asymmetrical "high heel" on the right and a sidelobe on the left (Figure 12(a)), possibly caused by optical cross-talk and spectral out-of-band leakage. (Band M2 has a similar feature but was not tested); 2) Band M11 DLSF has a trailing sidelobe (Figure 12(b)) at a magnitude of about 5\% at about two ASIs from the main lobe, possibly caused an internal optical reflection artifact. Because of these artifacts, a "waiver" MTF specification at 1.0 Nyquist frequency has been set to be greater than 0.29, as shown in the Figure 10.

For the I-bands HSR at the end of scan (EOS), the test results marginally exceed original specification for I-bands, particularly for bands I1 and I2 (Figure 11). The instrument EFL and non-ideal focus were speculated to be the root causes. A "wavier" was submitted against the HSR specification, loosening the performance compliance criteria. For the I-bands HSR at the nadir, the test results meet the specification (not shown here).
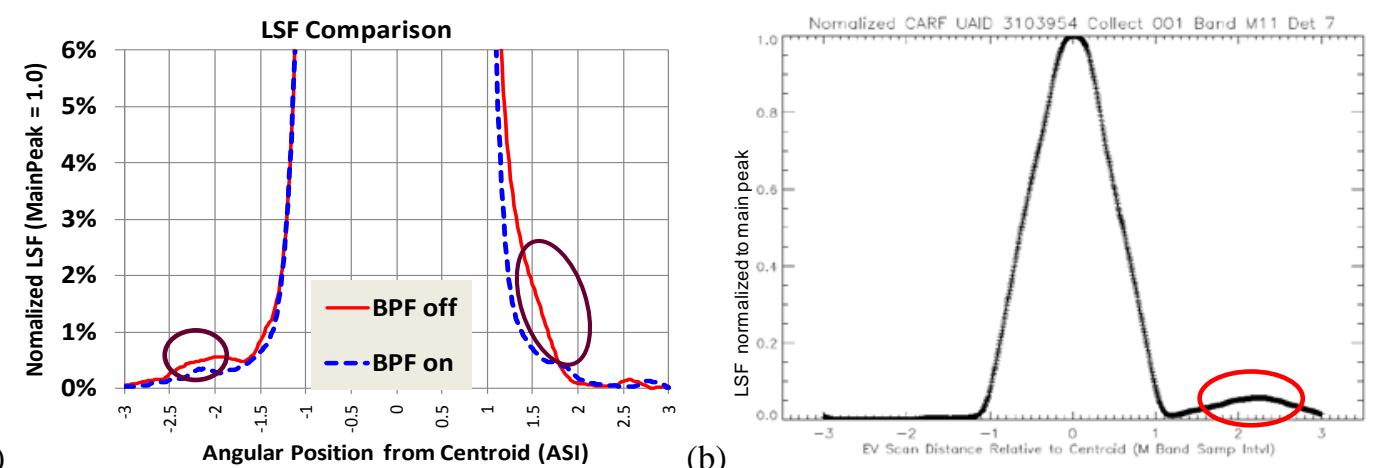

Figure 12. (a) Scan DLSF asymmetrical "high heel" on the right and sidelobe on the left in band M1; (b) Scan DLSF with a trailing sidelobe in band M11.

\section{SPCTRAL BAND-TO-BAND CO-REGISTRATION (BBR) PERFORMACE}

Since many VIIRS environmental data records (EDRs) are retrieved by using signals from multiple bands, it is crucial to have these bands co-registered between one another. The band-to-band co-registration (or "registration" as commonly called) (BBR) may be defined as the overlap of point spread functions (PSFs) from one detector in one spectral band to a corresponding detector in another spectral band. This may also be referred to as the detector-to-detector co-registration $(D D R)$. Mathematically, the $D D R$ between detector $i$ and detector $j$ may be expressed as 


$$
D D R_{i, j}=1-\oiint_{(x, y) \in R}\left|P S F_{i}-P S F_{j}\right| / 2 d x d y
$$

where $P S F$ is normalized, i.e., $\oiint_{(x, y) \in R} P S F_{k} d x d y=1$.

Since the actual PSF for each detector is not known a priori, for the purpose of assessing BBR from ground testing, each detector PSF is simplified as uniformly distributed over a pixel, which is largely true in the along-track direction. So in the sensor specification ${ }^{3}$, the above Eq. (6) is simplified as

$$
D D R_{i, j}=\left(1-\Delta S_{i, j}\right)\left(1-\Delta T_{i, j}\right)
$$

where $\Delta S_{i, j}$ and $\Delta T_{i, j}$ are the differences of DLOS and ILOS for the corresponding detectors in the scan and track directions, respectively. Their units are specified in $\mathrm{HSR}^{3}$ at the end of scan (EOS), which is close to the horizontal sampling interval (HSI) at the EOS. Since HSI is the projection of ASI on the earth surface, we use ASI throughout this paper. In the ground tests, as well as on-orbit, samples are sequentially recorded and transferred. Each increment in the sample sequence is exactly equivalent to an ASI. The telescope motion in ASI is exactly equivalent to the sample sequence. Thus it is more convenience to use the ASI directly in the process.

The sensor specification ${ }^{3}$ places the BBR requirement "at least $99.7 \%$ of corresponding pixel samples". We interpret this as the worst DDR between each band pair at the left/right edges of the scan in the non-aggregated zones. The specification is listed in the lower-left triangle in Figure 15.

The ground testing of DDR employs a similar principle as expressed in Eq. (2) but uses much wider reticle slits than those used in LSF testing. The purpose of using wider slits is to simultaneously acquire high signal-to-noise ratio (SNR) responses in as many bands as possible in one set of data, as was similarly done for MODIS ${ }^{7,8}$. For DDR in the track direction, a reticle with multiple staircase slits was used to obtain combined aperture response functions (CARFs) for all detectors in a band in one test dataset. The centroids of the CARFs yields spectral band registration (SBR) from a common reference. The results of apparent averaged band registration relative to band I1 from the TVac cold and nominal performance plateaus, and estimate from limited tests at TVac hot performance plateau are shown in Figure 13. As we can see, the SBR for bands in SWMWIR and LWIR FPAs changes less than 2\% M-band ASI from cold to nominal and to hot performance plateaus in TVac. They lead ahead of bands in VisNIR FPA in less than 5\% M-band ASI in the along-track direction.

In the scan direction, a reticle with multiple parallel slits perpendicular to the scan direction was used to obtain the CARFs. The results of apparent averaged band registration from the TVac cold and nominal performance plateaus, and estimate from limited tests at TVac hot performance plateau are shown in Figure 14. As we can see, the SBR for bands in SWMWIR changes about 3\% M-band ASI from cold to nominal and to hot performance plateaus in TVac. We also notice general trends of upward slope within each FPA, indicating that the bands on the trailing edges of the focal planes are behind those for the bands on the leading edges (Figures 14 and 2). This is because of a mismatch between the scan speed (too slow, but at the maximum allowed speed) and the instrument effective focal length (EFL), which is shorter than the specification.

Figures 13 and 14 show the band averaged $\Delta S_{i, j}$ and $\Delta T_{i, j}$ relative to those in band I1. Using Eq. (8), completed matrices of BBR are generated, as shown in the upper-right triangles in Figures 15 and 16. The lower-left triangle in Figure 15 shows the BBR specification, whereas the lower-left triangle in Figure 16 shows the number of detector pairs having DDR our-of-specification. As we can see, because of the shorter-than-specified EFL, BBR for band pairs M9/M13, two edge bands on the same FPA, is not compliant with the specification under all test conditions. The BBR for band pair M13/M14, one at the leading edge of the SWMWIR FPA and another on the trailing edge of the LWIR FPA, is non-compliant with the specification at the Cold Performance Plateau, the instrument temperature expected during the early on-orbit time period. The BBR for band pair I4/I5 is also non-compliant at the Cold Performance Plateau. Further scan timing adjustments can improve the BBR between bands M12/M13 and other bands.

For DDR/BBR between the I-bands and M-bands, a nesting scheme is used: two consecutive samples from an I-band in the scan direction from two adjacent detectors in the track direction are arithmetically averaged to form a sample from 
the so-formed "MI" detector from the I-band in radiometric response and their arithmetically averaged geometric center is to co-register with a corresponding detector in an M-band ${ }^{3}$. The BBR matrices between the "MI"-bands and M-bands are shown in the upper-right 5x17 rectangles in Figures 15 and 16.

\section{Band Avg Trk Reg relative to I1 in Cold and Nom Perf TV}

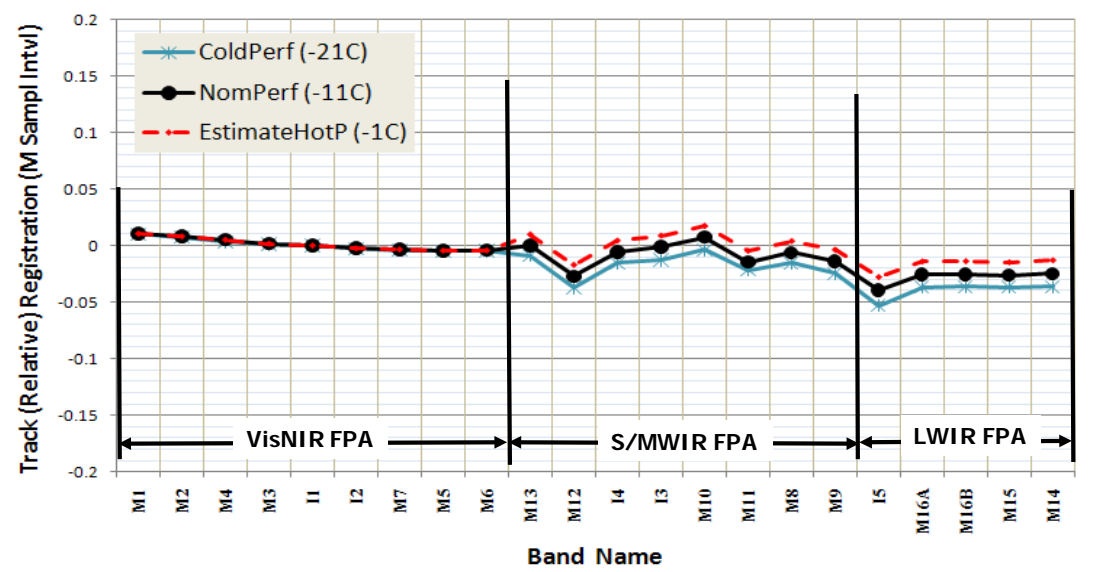

Figure 13. Results of averaged spectral band registration (SBR) in the track direction from the TVac cold and nominal performance plateaus, and estimate from limited tests at TVac hot performance plateau.

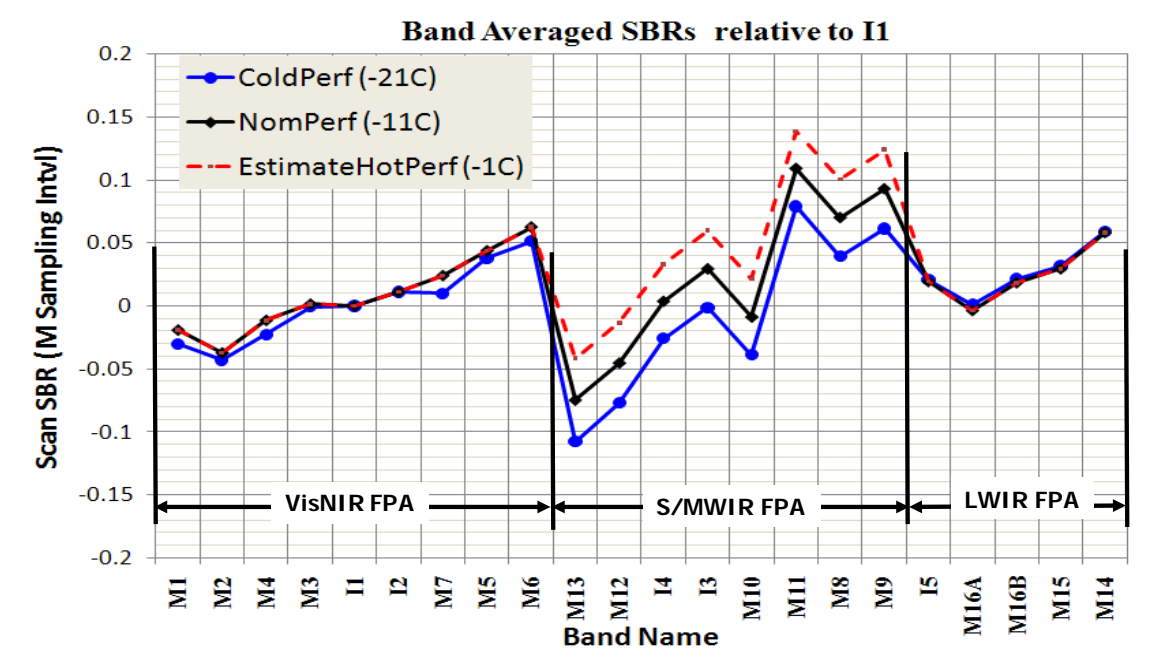

Figure 14. Averaged spectral band registration (SBR) in the scan direction from the TVac cold and nominal performance plateaus, and estimate from limited tests at the TVac hot performance plateau.

Note that BBR matrices in Figures 15 and 16 are the results from ground testing under "static", thermally soaked conditions. When the instrument is operated on-orbit, the BBR matrix may change due to a non-uniform thermal distribution that is dependent on orbital position. Other factors such as satellite launch shock, G-release, EFL change and scan speed variation may also affect on-orbit BBR performance. These factors may put a few other BBR pairs that have less than a 5\% margin (marked in yellow cells in Figures 15 and 16) out of specification. 


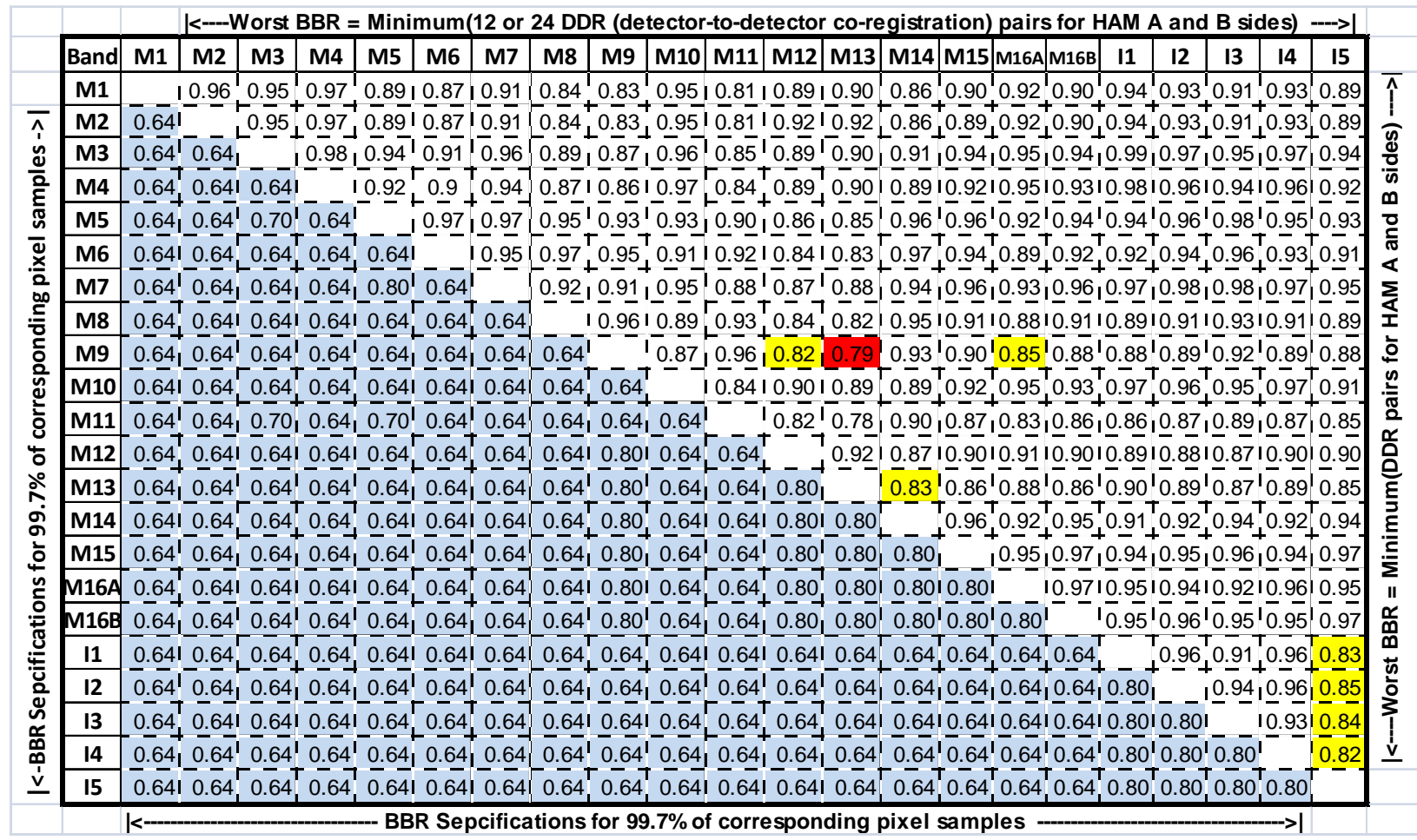

Figure 15. Static BBR results from the TVac nominal performance plateau.

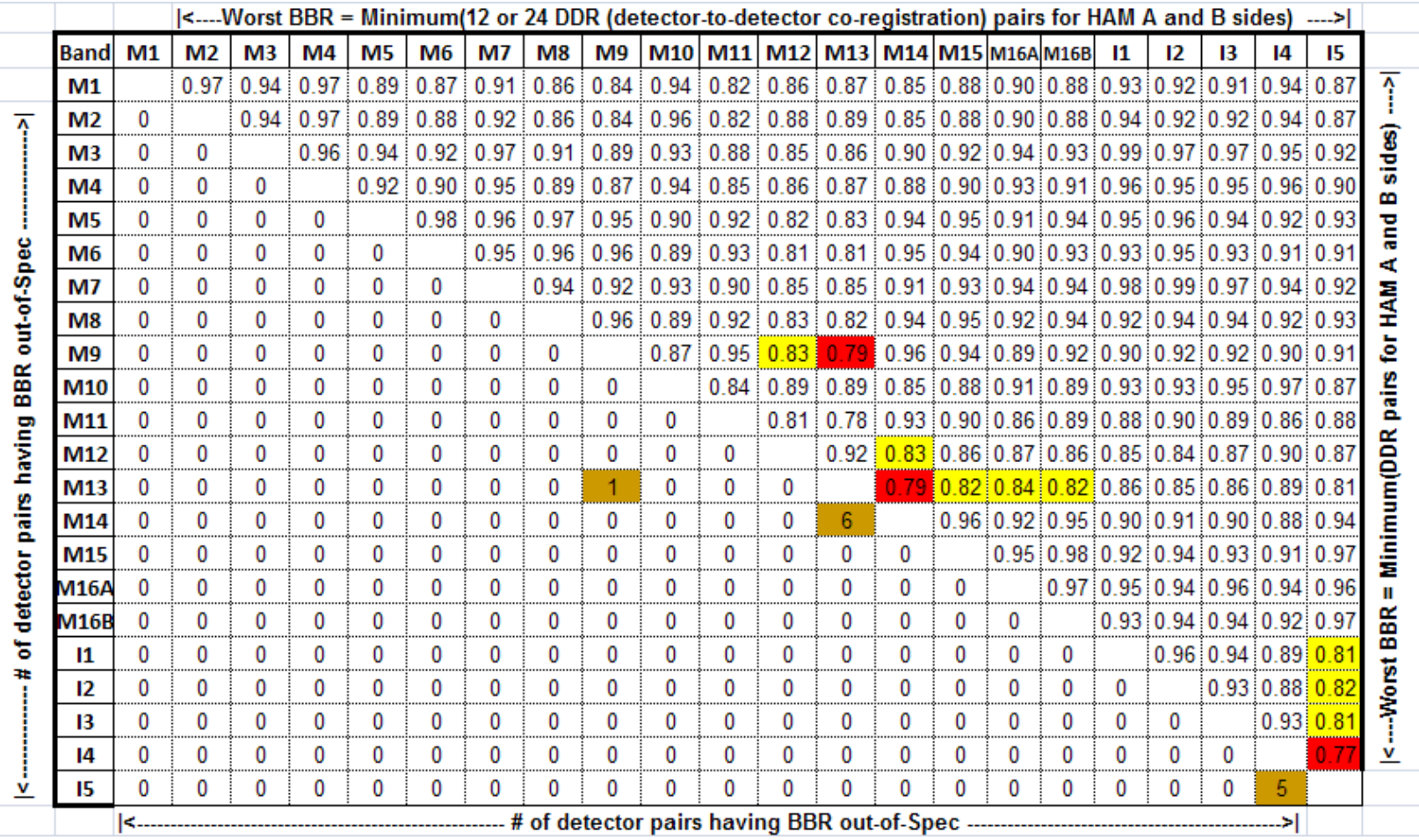

Figure 16. Static BBR results from the TVac cold performance plateau. 


\section{POINTING STABILITY PERFORMACE}

Pointing knowledge and stability is a concern to geolocation accuracy and precision. Sensor level pointing tests include measuring the scan center, scan plane tilt and pointing variations in the track and scan directions. Spacecraft level tests include sensor alignments relative to the spacecraft and attitude determination and control system (ADCS) reference frame. On-orbit pointing variations as functions of scan angle, orbit position and aging in the space environment are analyzed from structural, thermal and optical performance (STOP) modeling. Disturbance from the spacecraft, including other instruments, on the VIIRS also play a role in pointing variations.

Many factors affecting pointing, and thus geolocation, will be measured and corrected as necessary during early on-orbit intensive calibration and validation phase, and during long-term monitoring of the instrument. However, estimates of pointing errors and biases were made on the ground by measurements and modeling. Data measuring the scan center, scan plane tilt, instrument to spacecraft alignment has already been incorporated into the operational software. The operational software also uses the encoder data and timestamps from the RTA and HAM to pin-point geolocation in the scan direction, as shown in Figure 17, which shows the pointing deviation from average scan rate and average scan start bias.

Not yet incorporated in the operational software is the pointing variations caused by thermal distortion. The STOP model results show that lifetime on-orbit pointing stability in both scan and track directions, and one-day pointing stability in the scan direction are compliant with the specification. However, one-day pointing stability in the track direction is not compliant with the specification due to significant thermally induced structural displacement within an orbit, see Figure 18. The pointing is expected to change 30 arc-seconds in the scan direction and 45 arc-seconds in the track direction, which amounts to nadir ground displacements of $120 \mathrm{~m}$ and $180 \mathrm{~m}$, respectively. In this regard, a pointing correction that follows the orbital position is vital for accurate geolocation. Monitoring, analyzing, and correcting these errors are described in the following section.

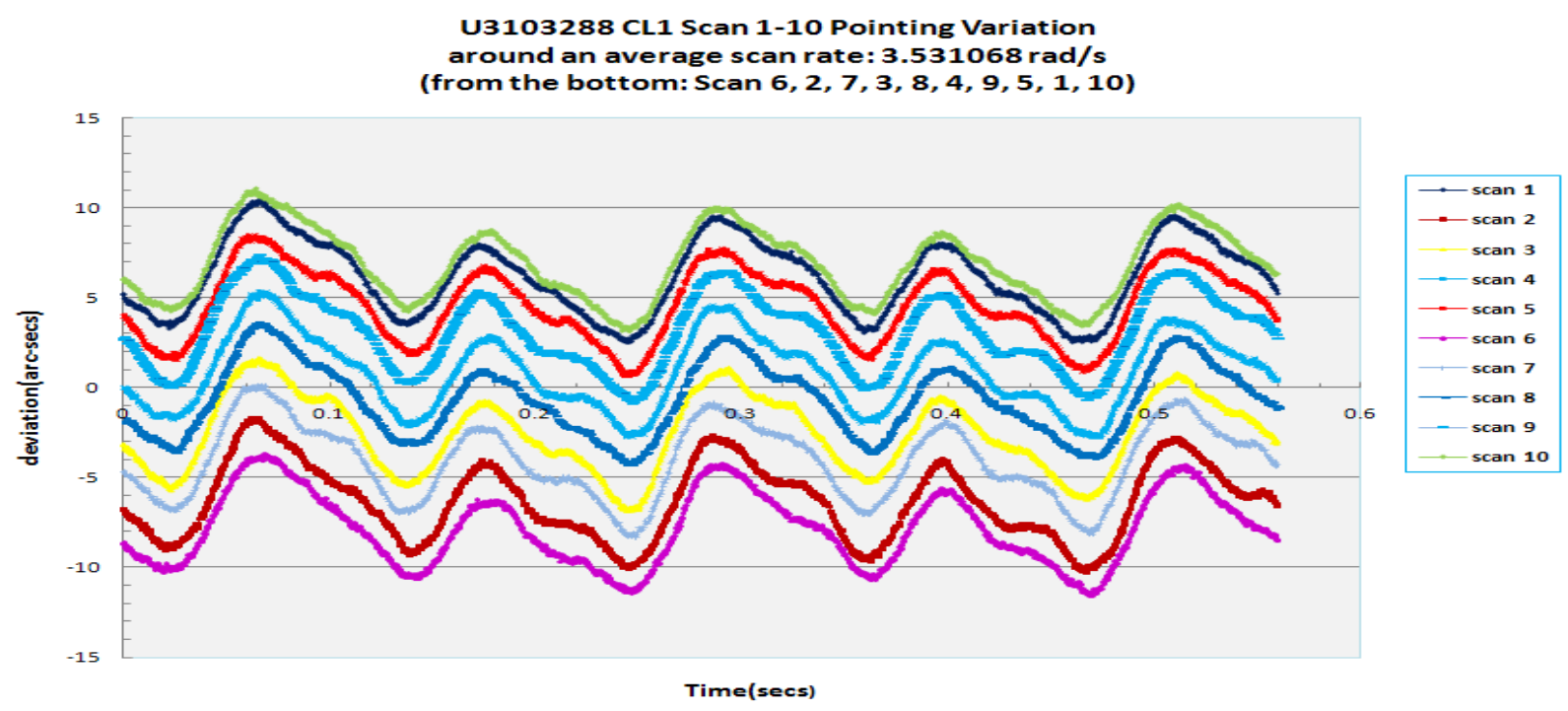

Figure 17. An example of pointing deviation from average scan rate and average bias in scan start positions. The curves will be followed closely with full complement of RTA/HAM encoder/timestamps information. This would be the pointing error in scan direction if the timestamps were lost (unlikely). 


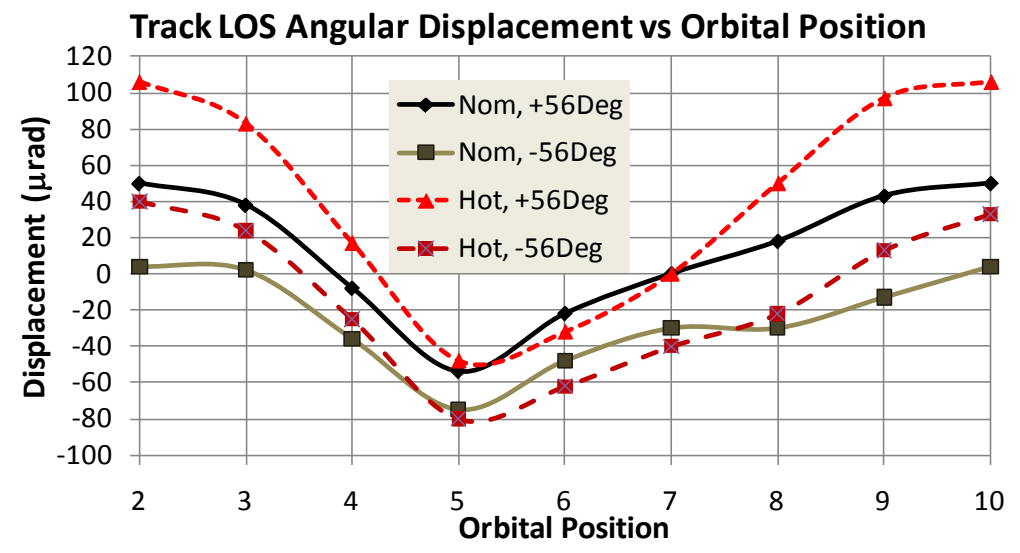

Figure 18. Examples of on-orbit track line-of-sight (LOS) pointing variation from STOP modeling at the nominal and hot performance plateaus for scan angles of $+56^{\circ}$ and $-56^{\circ}$.

\section{CONCLUDING REMARKS AND PATHS FORWARD}

The VIIRS instrument pre-launch tests and analysis of geometric performance have been completed. Overall, the performance parameters in spatial responses, band-to-band registration and pointing meet sensor specification, with some parameters for some bands/detectors marginally out-of-specification. Activities for on-orbit performance monitoring and/or corrections have been planned ${ }^{10,11}$ with tools developed and a majority of key tools rehearsed using test data.

On-orbit BBR performance will be monitored and fine tuned if necessary between band pairs by using lunar calibration data $^{12}$ and terrestrial edge features ${ }^{13}$. Special attention will be paid to those band pairs that already have out-ofspecification or low margin static BBR as shown in red and yellow cells in Figures 15 and 16. On-orbit sensor spatial response (LSF) performance can be estimated from earth scenes with linear features such as shorelines and long bridges. The detector LSFs can be derived from edge spread functions (ESFs) estimated from scenes with sharp edges. The lunar calibration data will also be useful for the spatial performance assessment ${ }^{14}$. Special attention will be paid to bands M1, M2 and M11 since their LSFs showed anomalous sidelobes or asymmetrical "high heels" in the ground tests.

The most important aspect of on-orbit geometric calibration and validation activities is the geolocation characterization and correction. Initial activities include: 1) verifying spacecraft (SC) auxiliary ephemeris (position and speed) and attitude data (roll, pitch and yaw); 2) verifying RTA/HAM encoder data for scan time, scan period, and scan rate stability (e.g., Figure 17); and 3) performing initial qualitative assessment of geolocation accuracy by overlaying VIIRS images with fine resolution Landsat scenes and then removing any large geolocation bias to reduce the residual errors.

A key activity in post-launch geolocation is to activate ground control point matching (CPM) program that has been used to achieve sub-pixel accuracies in MODIS ${ }^{15}$ and is being adapted for VIIRS. The CPM program utilizes over 1200 ground control point chips from finer resolution $(30 \mathrm{~m})$ Landsat images to cross-correlate against VIIRS imagery band images. The shifts of the CPM at maximum cross-correlation form a series of control point residuals. These residuals will be analyzed and the results will be used in the geolocation algorithm lookup table (LUT) for corrections of various sensor parameters such as instrument mounting, attitude changes, "diurnal" sun angle variations as a proxy of thermal variations (e.g. Figure 18), scan angle variations, seasonal cycles, and long term trends. A lately developed Kalman filtering technique will be used (first by testing) to enable nearly autonomous long-term trending of geolocation data, thus potentially making the long-term error analysis operational for VIIRS ${ }^{16}$.

\section{ACKNOWLEDGEMENTS}

The authors acknowledge the sponsorship of this work by NASA NPP Science Data Segment (SDS), and the cooperation and assistance from many colleagues from Raytheon Company, Northrop Grumman Aerospace Systems, the NPOESS Integrated Program Office (IPO), The Aerospace Corporation, MIT Lincoln Lab, NASA NPP VIIRS Ocean 
Science Team (VOST), NASA NPP Instrument Calibration and Support Team (NICST), the NPP Instrument Calibration and Support Element (NICSE), and NASA NPP Project Science Office.

\section{REFERENCES}

[1] Weiss, Stephanie (2011), VIIRS Geolocation Algorithm Theoretical Basis Document ATBD (ref Y3258), (CDRL A032, Document Number D43776], document Date: 25 March 2011, Revision: D2)”, Northrop Grumman Aerospace Systems, Redondo Beach, CA.

[2] Mills, Steve (2010), VIIRS Radiometric Calibration Algorithm Theoretical Basis Document ATBD (ref Y3261), (Document Number D43777, document Date: 30 June 2010, Revision: F)”, Northrop Grumman Aerospace Systems, Redondo Beach, CA.

[3] Performance Specification Sensor Specification for the Visible/Infrared Imager Radiometer Suite (VIIRS), PS154640-101E, Raytheon, El Segundo, CA, 21 Nov, 2009.

[4] Fang, D.T and Puschell, J. (2010), "Imagery spatial performance throughput correction methodology," Remote Sensing System Engineering III, SPIE 7813, doi: 10.1117/12.860740.

[5] Oppenheim, A.V. and R.W. Schafer, 1989: [Discrete-time Signal Processing]. Prentice Hall, New Jersey, 879 pp.

[6] http://en.wikipedia.org/wiki/Convolution

[7] Montgomery, H., N. Che, and J. Bowser, "Determination of the spatial characteristic by using the Spectro-

Radiometric Calibration Assembly (SRCA) of MODIS (Part I. Along-scan)”, Proc. of SPIE, Vol. 3439, San Diego, 1998, pp.226-237.

[8] Montgomery, H., N. Che, and J. Bowser, "Determination of the spatial characteristic by using the SpectroRadiometric Calibration Assembly (SRCA) of MODIS (Part II. Along-track)”, Proc. of SPIE, Vol. 3439, San Diego, 1998, pp.238-246

[9] Xiong, X., N. Che, W. Barnes, Y. Xie, L. Want, and J. Wu. (2006) "Status of Aqua MODIS Spatial Characterization and Performance”, Proc. of SPIE, Vol. 6361, doi: 10.1117/12.687162

[10] Visible/Infrared Imager/Radiometer Suite (VIIRS) Sensor Data Record (SDR) Calibration and Validation Plan for NPP, D47854-01, Northrop Grumman Space Technology, Redondo Beach, CA, 0324 March, 2009.

[11] DeLuccia, F. J. and Cao, C (2011), VIIRS SDR Calibration/Validation Operations Concept (OPSCON) Document, V1.0, 16 May 2011.

[12] Xiong, X., J. Sun, S. Xiong, W. L. Barnes (2004), “Using the Moon for MODIS On-orbit Spatial Characterization”, Editors: R. Meynart, S. P. Neeck, H. Shimoda, J. B. Lurie and M. L. Aten, "Sensors, Systems, and Next-Generation Satellites VII”, Proceedings of SPIE, Vol. 5234.

[13] Yang, K., A. J. Fleig, R. E. Wolfe, and M. Nishihama (2000), “MODIS band-to-band registration”, Proceedings of IGARSS, 2000, pp.887-889.

[14] Wang, Zhipeng, Choi, T. J., and Xiong, X. (2011), "Using the moon for Terra MODIS MTF characterization”, Proceedings of SPIE, this issue, in press.

[15] Wolfe, R. E., and M. Nishihama (2009), “Trends in MODIS geolocation error analysis”, Editors: Butler, J. J., X. Xiaoxiong, G. Xingfa, Earth Observing Systems XIV, Proceedings of SPIE, Vol. 7452, 74520L, doi: 10.1117/12.826598.

[16] Nishihama, M., R.E. Robert (2011), "MODIS and VIIRS Geolocation Error and Long-term Trend Analysis with Automated Correction Techniques using Kalman Filtering”, Proceedings of IGARSS, July 24-29, 2011, Vancouver, Canada, in press. 